\title{
Meson masses and decay constants from unquenched lattice QCD
}

\author{
K. Jansen, ${ }^{1}$ C. McNeile, ${ }^{2}$ C. Michael, ${ }^{3}$ and C. Urbach ${ }^{4}$ \\ ${ }^{1}$ DESY, Zeuthen, Platanenallee 6, D-15738 Zeuthen, Germany* \\ ${ }^{2}$ Department of Physics and Astronomy, The Kelvin Building, University of Glasgow, Glasgow, G12 8QQ, United Kingdom \\ ${ }^{3}$ Theoretical Physics Division, Department of Mathematical Sciences, University of Liverpool, Liverpool L69 3BX, United Kingdom ${ }^{\ddagger}$ \\ ${ }^{4}$ Humboldt-Universität zu Berlin, Institut für Physik Mathematisch-Naturwissenschaftliche Fakultät I, \\ Theorie der Elementarteilchen/Phänomenologie, Newtonstraße 15, 12489 Berlin Germany ${ }^{\S}$
}

(Received 2 July 2009; published 29 September 2009)

\begin{abstract}
We report results for the masses of the flavor nonsinglet light $0^{++}, 1^{--}$, and $1^{+-}$mesons from unquenched lattice QCD at two lattice spacings. The twisted mass formalism was used with two flavors of sea quarks. For the $0^{++}$and $1^{+-}$mesons we look for the effect of decays on the mass dependence. For the light vector mesons we study the chiral extrapolations of the mass. We report results for the leptonic and transverse decay constants of the $\rho$ meson. We test the mass dependence of the KSRF relations, between the mass, leptonic coupling constant, and strong coupling of the rho meson.
\end{abstract}

DOI: 10.1103/PhysRevD.80.054510

\section{INTRODUCTION}

Modern unquenched lattice QCD calculations include the dynamics of light sea quarks (with pion masses below $300 \mathrm{MeV}$ ) and use multiple lattice spacings and volumes [1]. This has allowed calculations of many basic quantities of long-lived hadrons that decay via the weak force to be computed to high accuracy. Of particular note is that unquenched lattice QCD calculations are now making contact with the results of chiral perturbation theory calculations $[2,3]$, particularly for light pseudoscalar mesons.

There has been much less work on studying resonances with the latest generation of lattice QCD calculations. Some of the most interesting questions in light quark hadron spectroscopy are looking for glueball degrees of freedom in the experimental $f_{0}$ mesons and looking for experimental evidence for the exotic $1^{-+}$mesons. There are new experiments, such as Gluex [4] and PANDA [5] that will start around 2015, that aim to study hadronic resonances. The new hadronic physics experiments will require results from lattice QCD to guide their searches for new hadrons. The lattice results for light resonances have recently been reviewed by [6-11].

In this paper we test basic lattice QCD techniques to study the $b_{1}, a_{0}$, and $\rho$ mesons. The observation of the decay of the $\rho$ meson has been a long goal of the lattice community. The issue of dealing with the decay of the $\rho$ meson has stopped many calculations of weak decays such as $B \rightarrow \rho \nu e$ [12]. In the case of determining $\left|V_{u b}\right|$ from the semileptonic decay $B \rightarrow \rho \nu e$, the simplest thing is to just ignore this decay and focus on $B \rightarrow \pi \nu e$. However there are some very important reactions such as $B \rightarrow K^{\star} \gamma$ and $B \rightarrow \rho \gamma$ that have no simple equivalent form factors with a meson that is stable under strong decay. The effect of the

\footnotetext{
*karl.jansen@desy.de

†c.mcneile@physics.gla.ac.uk

*cmi@liverpool.ac.uk

§Carsten.Urbach@physik.hu-berlin.de
}

PACS numbers: 11.15.Ha, 12.38.Gc, 14.40.Cs

strong decays on these lattice calculations is an unknown systematic error. It is also important to understand the effect of strong decay on the $\rho$ meson for calculations relevant to $g-2[13,14]$.

It has been proposed (see [6-9] for a review) that the $a_{0}(980)$ contains tetraquark or molecular degrees of freedom. It is interesting to see whether quark-antiquark operators actually couple to this state in lattice QCD calculations. Understanding whether the $a_{0}(980)$ is a tetraquark is important for classifying the $f_{0}$ and $a_{0}$ mesons into $\bar{q} q$ or $\overline{q q} q q$ multiplets [9].

First we define some notation. We call the lightest flavor nonsinglet states from the lattice calculations with $J^{P C}$ given by $0^{++}, 1^{+-}$, and $1^{--}$as the $a_{0}, b_{1}$, and $\rho$ mesons, respectively, at the masses used in the lattice calculation. We include the mass of the state when we deal with the experimental state, such as $a_{0}(980), \rho(770)$.

The plan of the paper is thus. We first discuss some general issues about the effect of hadronic decays on mesons. We then describe the details of the lattice QCD calculation and report results for the masses in lattice units. In Sec. IV we discuss the interpretation of the results for the $a_{0}$ and $b_{1}$ channels. In Sec. V we then discuss the results for the masses of the vector mesons. We then discuss the leptonic decay constant of the $\rho$ meson. In the penultimate section we test the KSRF relations. In Sec. VIII we draw our conclusions.

\section{GENERIC BACKGROUND TO THE CALCULATION}

At first analysis, it is not clear that the concept of a hadronic resonance makes sense in an Euclidean lattice QCD calculation with a finite box size. Naively, the size of the decay width could be a measure of the systematic error on the mass of the resonance on the lattice, however there are arguments that suggest this is a pessimistic estimate. Michael [15] reviews some of the phenomenology of unstable hadrons and notes that many unstable mesons fit 
well with mesons that are stable under the strong decays, using SU(3) symmetry, for example. Also Bijnens et al. [16] obtained acceptable fits to the masses of the light vector mesons with an effective theory (but some parameters coming from a model) that did not include the effect of the vector meson decay.

In Fig. 1 we show a "picture" of what we expect happens when a resonance $(R)$ decays to two mesons $M_{1}$ and $M_{2}$ in the lattice calculation. When the mass of the decay channels and the resonance are close there is mixing between them (an avoided level crossing). The hadronic decay in Fig. 1 requires the creation of a quark- antiquark pair, so it is only present in unquenched lattice QCD calculations.

For an $S$-wave decay the threshold for decay is $M_{R}=$ $M_{1}+M_{2}$. For a $P$-wave decay the decay products must carry momentum. For example, in the real world the $\rho$ decays into two pions, via a $P$-wave decay. The threshold for decay at rest is $2 \sqrt{m_{\pi}^{2}+\left(\frac{2 \pi}{L}\right)^{2}}$ where $L$ is the side of the box, assuming periodic boundary conditions in space. The CERN group $[17,18]$ found excited masses for the $\rho$ channel that were consistent with $2 \sqrt{m_{\pi}^{2}+\left(\frac{2 \pi}{L}\right)^{2}}$. For heavy quark masses it can be more kinematically favorable to study the decay of the $\rho$ meson with one unit of momentum to decay to a pion at rest and a pion with one unit of momentum [19-21]. It may well be that one of the mesons $\left(M_{1}\right.$ or $\left.M_{2}\right)$ in Fig. 1 is also a resonance, in that case there will be second decay. One example of this is one of the decays of the $b_{1}$ meson.

$$
b_{1} \rightarrow \omega \pi \rightarrow \pi(\pi \pi \pi)
$$

Our lattice calculations can in principle test the effect of the opening of decay thresholds, because as we lower the sea quark masses in the calculations, the various decays channels become open. In practice it may be hard to see the effect of the open decay as the quark mass changes, because other systematic errors may change as well.

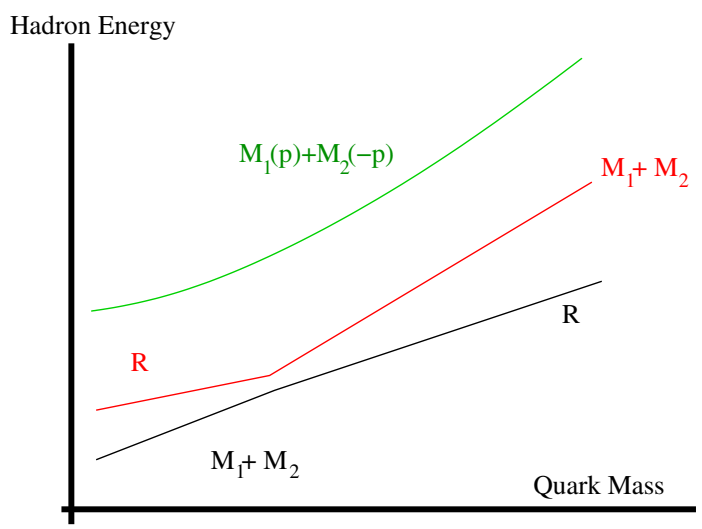

FIG. 1 (color online). The effect of decays on the energy levels of a resonance at finite volume.
Although it appears that $S$-wave decays are kinematically easier to observe than $P$-wave decays, the $a_{0}$ and $b_{1}$ mesons are noisier than the $\rho$ meson. The $\rho$ meson at rest is stable to two-pion decay in this calculation, so for this state we try to build in the physics of the meson decay by studying the chiral extrapolation formulas in Sec. V. We also estimate the decay transition amplitude directly on the lattice, to gain an understanding of possible consequences of the mixing of the $\rho$ meson with the two-pion state.

The MILC Collaboration claimed to see some evidence for the $a_{0}$ resonance to decay into two light hadrons [22]. Latter work showed that more analysis was required to understand the $a_{0}$ decay in staggered calculations [23-25].

Lüscher has developed a technique to compute the scattering phase shifts [26]. The method was applied to 2-d theories [27] and the $\phi^{4}$ theory [28]. We have not investigated newer methods [29,30] based on Lüscher's technique [26], but plan to do so in the near future. Morningstar [31] has recently presented a simple example of the basic method in quantum mechanics [32].

\section{DETAILS OF THE LATTICE CALCULATION}

Our lattice calculation uses the twisted mass QCD formalism [33]. Once a single parameter has been tuned, twisted mass QCD has nonperturbative $O(a)$ improvement [34]. We call this maximally twisted mass QCD (MTMQCD). This $O(a)$ improvement was checked numerically by scaling studies using quenched QCD calculations [35-39], and has recently been checked in lattice perturbation theory [40]. As a prerequisite for large-scale unquenched calculations, the phase structure of twisted mass QCD has been studied [41-45]. The twisted mass formalism has recently been reviewed by Shindler [46].

The ETM Collaboration has already published a comparison of the lattice results for $m_{\pi}$ and $f_{\pi}$ against chiral perturbation theory $[47,48]$. Results for the nucleon and $\Delta$ masses and a comparison with chiral perturbation theory are reported in [49]. The masses of the flavor singlet pseudoscalar mesons have been presented [50]. Light quark masses and decay constants from a partially quenched analysis have been published from this data set [51]. There are ongoing projects to look at the moments of parton distributions [52,53], the form factor of the pion [54], and the properties of heavy-light mesons [55]. For an overview of the broad range of physics projects undertaken by the ETM Collaboration see the review by Urbach [56].

For the gauge fields we use the tree-level Symanzik improved gauge action [57], which includes the plaquette term $U_{x, \mu, \nu}^{1 \times 1}$ and rectangular $(1 \times 2)$ Wilson loops $U_{x, \mu, \nu}^{1 \times 2}$

$$
\begin{aligned}
S_{g}= & \frac{\beta}{3} \sum_{x}\left(b_{0} \sum_{\substack{\mu, \nu=1 \\
1 \leq \mu<\nu}}^{4}\left\{1-\operatorname{retr}\left(U_{x, \mu, \nu}^{1 \times 1}\right)\right\}\right. \\
& \left.+b_{1} \sum_{\substack{\mu, \nu=1 \\
\mu \neq \nu}}^{4}\left\{1-\operatorname{retr}\left(U_{x, \mu, \nu}^{1 \times 2}\right)\right\}\right)
\end{aligned}
$$


with $b_{1}=-1 / 12$ and $b_{0}=1-8 b_{1}$. This choice of gauge action was made after a study of the phase structure of unquenched QCD with $n_{f}=2$ mesons.

The fermionic action for two degenerate flavors of quarks in twisted mass QCD is given by

$$
S_{F}=a^{4} \sum_{x} \bar{\chi}(x)\left(D_{W}[U]+m_{0}+i \mu \gamma_{5} \tau^{3}\right) \chi(x)
$$

with $\tau^{3}$ the Pauli matrix acting in the isospin space, $\mu$ the bare twisted mass and the massless Wilson-Dirac operator given by

$$
D_{W}[U]=\frac{1}{2} \gamma_{\mu}\left(\nabla_{\mu}+\nabla_{\mu}^{*}\right)-\frac{a r}{2} \nabla_{\mu} \nabla_{\mu}^{*} .
$$

where

$\nabla_{\mu} \psi(x)=\frac{1}{a}\left[U_{\mu}^{\dagger}(x) \psi(x+a \hat{\mu})-\psi(x)\right]$ and

$\nabla_{\mu}^{*} \psi(x)=-\frac{1}{a}\left[U_{\mu}(x-a \hat{\mu}) \psi(x-a \hat{\mu})-\psi(x)\right]$.

Maximally twisted Wilson quarks are obtained by setting the untwisted quark mass $m_{0}$ to its critical value $m_{\mathrm{cr}}$, while the twisted quark mass parameter $\mu$ is kept nonvanishing in order to work away from the chiral limit. In Eq. (3) the quark fields $\chi$ are in the so-called "twisted basis." The "physical basis" is obtained for maximal twist by the simple transformation

$$
\begin{aligned}
& \psi(x)=\exp \left(\frac{i \pi}{4} \gamma_{5} \tau^{3}\right) \chi(x), \\
& \bar{\psi}(x)=\bar{\chi}(x) \exp \left(\frac{i \pi}{4} \gamma_{5} \tau^{3}\right) .
\end{aligned}
$$

In terms of the physical fields the action is given by

$$
\begin{aligned}
S_{F}^{\psi}= & a^{4} \sum_{x} \bar{\psi}(x)\left(\frac{1}{2} \gamma_{\mu}\left[\nabla_{\mu}+\nabla_{\mu}^{*}\right]\right. \\
& \left.-i \gamma_{5} \tau^{3}\left(-\frac{a r}{2} \nabla_{\mu} \nabla_{\mu}^{*}+m_{\mathrm{cr}}\right)+\mu\right) \psi(x) .
\end{aligned}
$$

The generation of the gauge configurations is reported in $[48,58,59]$. The methods used to compute the correlators and extract the masses and decay constants of the light mesons, from $n_{f}=2$ unquenched twisted mass QCD are described in $[47,48]$. Reference [48] is intended to be the main reference that explains the basic lattice techniques used by the ETM Collaboration, such as those used to compute the light meson spectrum. Here we briefly summarize some of the methods from [48]. We used meson interpolating operators in the twisted basis (based on Eq. (6)), as described in [48]. The ensembles used in this calculation are summarized in Table I. Correlators separated by 10 trajectories were used. We fit a matrix of correlators to a factorizing fit form [48]. Correlated fits with reasonable $\chi^{2} /$ dof were used to choose the fitting regions in time. The basis of smearing functions includes
TABLE I. Summary of ensembles used in this calculation. The format of the measurement column is number of blocks times block length.

\begin{tabular}{lclcc}
\hline \hline Ensemble & $\beta$ & $\mu$ & $L^{3} \times T$ & Measurements \\
\hline$B_{1}$ & 3.9 & 0.004 & $24^{3} \times 48$ & $111 \times 8$ \\
$B_{2}$ & 3.9 & 0.0064 & $24^{3} \times 48$ & $78 \times 32$ \\
$B_{3}$ & 3.9 & 0.0085 & $24^{3} \times 48$ & $66 \times 32$ \\
$B_{4}$ & 3.9 & 0.01 & $24^{3} \times 48$ & $38 \times 32$ \\
$B_{5}$ & 3.9 & 0.015 & $24^{3} \times 48$ & $44 \times 32$ \\
$B_{6}$ & 3.9 & 0.004 & $32^{3} \times 64$ & $81 \times 6$ \\
$C_{1}$ & 4.05 & 0.003 & $32^{3} \times 64$ & $64 \times 8$ \\
$C_{2}$ & 4.05 & 0.006 & $32^{3} \times 64$ & $66 \times 8$ \\
$C_{3}$ & 4.05 & 0.008 & $32^{3} \times 64$ & $61 \times 8$ \\
$C_{4}$ & 4.05 & 0.012 & $32^{3} \times 64$ & $40 \times 8$ \\
\hline \hline
\end{tabular}

local and fuzzed operators. A fuzzing length of 6 in lattice units was used [48].

The correlators were calculated with all-to-all quark propagators computed using the "one-end-trick" $[48,60]$. The all-to-all propagators used $Z_{2}$ noise in both the real and complex components from a single time source, chosen randomly for each measurement to reduce the autocorrelations [48]. A variant of "spin-dilution" is used to compute the all-to-all propagators called "linked-sources" [48], so that all 16 local quark-antiquark bilinears were constructed.

At finite lattice spacing, there is an order $a$ mixing of mesons with different parity in MTMQCD. When studying charged mesons this has the consequence that the $\rho$ and $a_{1}$ mesons mix. Assuming we are at maximal twist, the mixing will be of order $a$, then at large $t$ the lightest state, the $\rho$ meson, will dominate. The $\rho$ can be created by a vector or tensor current so we used a 4 by 4 matrix of correlators (vector/tensor and local/fuzzed). We obtain a good fit with one meson state for the time regions $8-18$ and 10-20 for $\beta$ 3.9 and 4.05 , respectively. We checked these fits using either a subset of operators or with more states.

The charged $a_{0}$ and $b_{1}$ mesons mix under twisting with spin-exotic mesons so we do not expect at large $t$ any significant contributions from parity mixing since those states will be heavy. For these cases, we fit a 2 by 2 matrix of correlators (local/fuzzed) with two meson states, using the fit regions in time $4-12$ and $5-14$, for $\beta=3.9$ and 4.05 , respectively.

In Table II we report the masses for the $a_{0}, b_{1}$ and $\rho$ mesons in lattice units. In Fig. 2 we plot the effective mass plot for the $\rho$ correlators for the $B_{6}$ ensemble.

In Sec. IV we process the raw data and convert the results into physical units. To convert the results into lattice units we use the scale from the pion decay constant, at $a_{\beta=3.9}=0.0855(5) \mathrm{fm}$ and $a_{\beta=4.05}=0.0667(5) \mathrm{fm}$. These scales were consistent with those obtained from the mass of the nucleon [49].

In Table II we also include the lattice masses for the neutral $\rho^{0}$ operator. In the twisted mass formalism the $\rho^{0}$ 
TABLE II. Masses in lattice units for the $a_{0}, b_{1}$, and $\rho$ mesons.

\begin{tabular}{lcccc}
\hline \hline Ensemble & $a m_{b_{1}}$ & $a m_{a_{0}}$ & $a m_{\rho^{+}}$ & $a m_{\rho^{0}}$ \\
\hline$B_{1}$ & $0.702(52)$ & $0.539(115)$ & $0.404(22)$ & $0.391(17)$ \\
$B_{2}$ & $0.685(28)$ & $0.573(59)$ & $0.422(9)$ & $0.434(17)$ \\
$B_{3}$ & $0.729(24)$ & $0.619(31)$ & $0.428(8)$ & $0.424(14)$ \\
$B_{4}$ & $0.681(29)$ & $0.666(34)$ & $0.438(6)$ & $\ldots$ \\
$B_{5}$ & $0.746(30)$ & $0.699(28)$ & $0.481(7)$ & $\ldots$ \\
$B_{6}$ & $0.674(29)$ & $0.636(53)$ & $0.416(14)$ & $0.409(21)$ \\
$C_{1}$ & $0.552(38)$ & $0.509(45)$ & $0.335(12)$ & $0.352(23)$ \\
$C_{2}$ & $0.555(29)$ & $0.410(29)$ & $0.337(12)$ & $0.344(13)$ \\
$C_{3}$ & $0.526(35)$ & $0.511(26)$ & $0.345(8)$ & $\cdots$ \\
$C_{4}$ & $0.638(32)$ & $0.545(19)$ & $0.368(6)$ & $\cdots$ \\
\hline \hline
\end{tabular}

and $\rho^{+}$mesons are not degenerate because of the flavor violation from the twisted mass term. The results in Table II show that the $\rho^{0}$ and $\rho^{+}$are essentially degenerate. A theoretical discussion with numerical examples for why this is so is contained in [61].

As reported in [61] the main effect of the flavor violation from the twisted mass term is in the mass splitting between the mass of the $\pi^{0}$ and $\pi^{+}$mesons. This has implications for decay thresholds of the $\rho^{+}$and $\rho^{0}$ mesons. Experimentally the dominant decays of the $\rho^{+}$and $\rho^{0}$ meson are to $\pi^{+} \pi^{0}$ and $\pi^{+} \pi^{-}$, respectively. The physical decay of $\rho^{0}$ to $\pi^{0} \pi^{0}$ is not allowed, because of isospin symmetry, however at nonzero lattice spacing this decay is allowed in twisted mass lattice QCD. At $\beta=3.9$ the mass splitting between the $\pi^{0}$ and $\pi^{+}$is approximately $50 \mathrm{MeV}$ at $\mu=0.004$ [48]. This should be compared with one unit

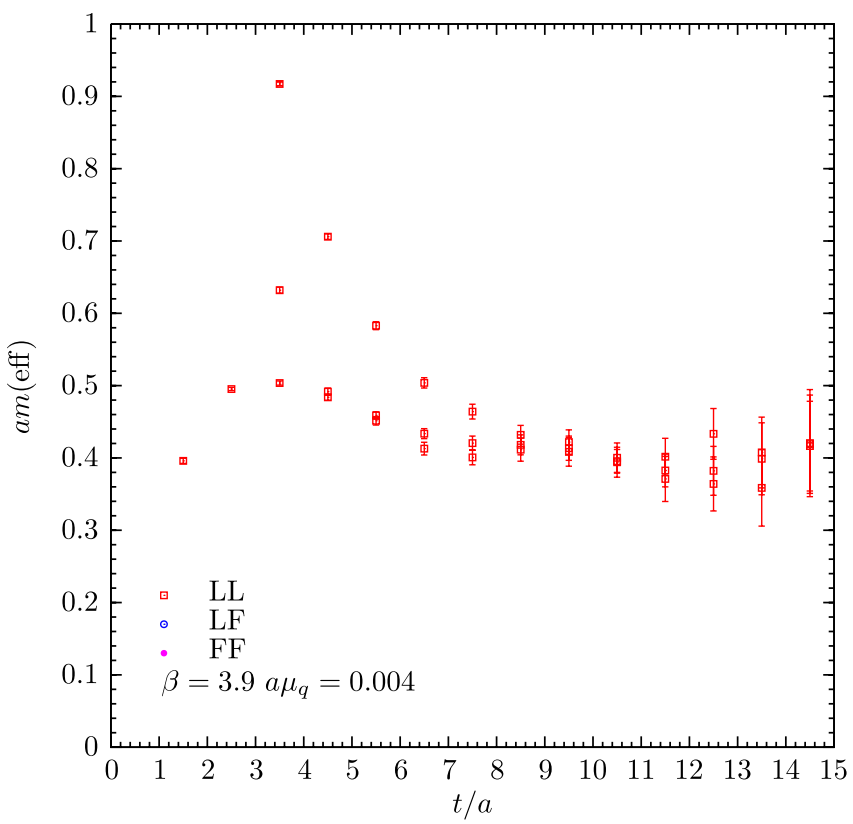

FIG. 2 (color online). Effective mass plot for the charged $\rho$ correlators (vector coupling) for the $B_{6}$ ensemble. $F$ and $L$ are the fuzzed and local operators, respectively. of quantized momentum of $600 \mathrm{MeV}$ and $450 \mathrm{MeV}$ on the $24^{3}$ and $32^{3}$ lattices, respectively, at $\beta=3.9$.

For a study of flavor singlet vector mesons such as the $\phi$ and $\omega$, evaluation of disconnected diagrams is required. Earlier lattice work [62] showed that these contributions are small. For the tensor coupling of the vector meson, the considerable variance reduction possible using MTMQCD has allowed these contributions to be evaluated with some precision for the first time [63] so yielding first principles results on the $\omega-\rho$ mass difference and mixing.

Here we are discussing the flavor nonsinglet mesons. For the neutral $\rho$ meson there are also disconnected diagrams that contribute to the correlators, because the twisted mass formalism breaks isospin symmetry at nonzero lattice spacing. These contributions would be expected to be small but, to check this, for the $B_{1}$ and $C_{1}$ ensembles we computed the relevant disconnected diagram for the vector mesons. Because of favorable variance reduction [48], we are able to determine the disconnected contribution rather precisely for neutral $\rho$ correlations using a vector coupling. The results are in Figs. 3 and 4. As we explain in Sec. VI, the neutral and charged vector currents renormalize differently, thus explaining that the ratio of correlators tends to something close to 2, rather than 1. The neutral and charged tensor current renormalize the same way, so the ratio of correlators is close to 1 . For both ensembles the disconnected diagrams make a negligible contribution to the correlators, so we do not consider their contribution any further.

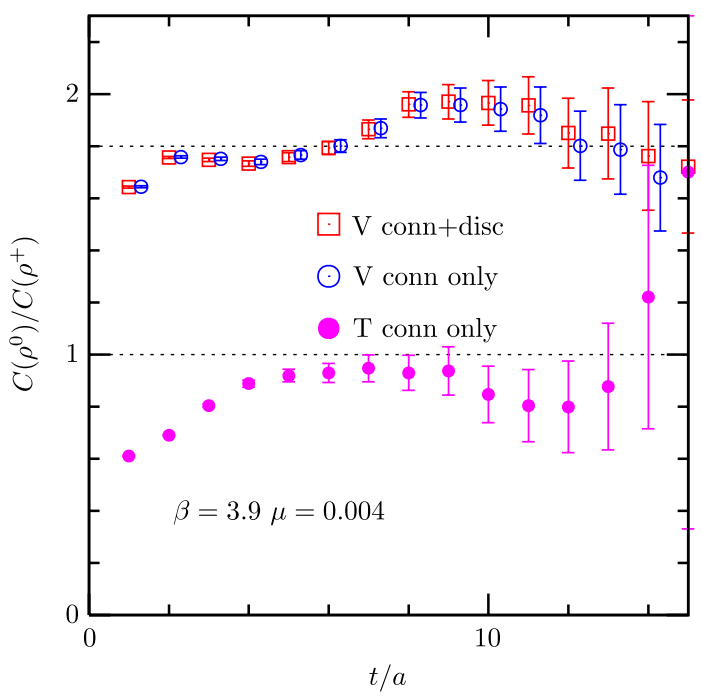

FIG. 3 (color online). The ratio of correlators for the (i) connected neutral and (ii) connected plus disconnected neutral to the connected charged $\rho$ correlator for the $B_{1}$ ensemble. $T$ is the tensor and $V$ is the vector current. As discussed in Sec. VI the different currents renormalize differently, which explains whether the ratio tends to one or to the ratio of the square of the renormalization factors. 


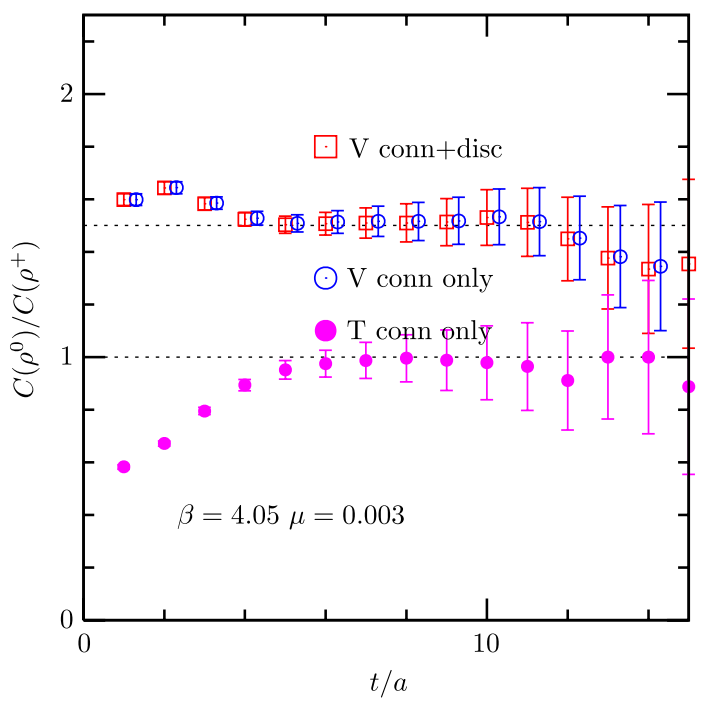

FIG. 4 (color online). The ratio of correlators for the (i) connected neutral and (ii) connected plus disconnected neutral to the connected charged $\rho$ correlator for the $C_{1}$ ensemble. The notation is the same as for the caption of Fig. 3.

\section{RESULTS FOR THE MASSES OF THE $a_{0}$ AND $b_{1}$ MESONS}

The results for the mass of the lightest flavor singlet $0^{++}$ meson from lattice QCD up to 2007 have been reviewed [6-8]. The physics goal is to decide whether a $\bar{q} q$ interpolating operator will couple to the experimental $a_{0}(980)$. The basic summary of the older quenched work was that $\bar{q} q$ interpolating operators did not see the $a_{0}(980)$ meson and coupled to the higher nonsinglet state.

The unquenched calculation by the RBC Collaboration [64] using $n_{f}=2$ domain wall fermions also found a mass close to the mass of the experimental state $a_{0}(1450)$. In an update on their analysis, that included 5 times the statistics, the RBC Collaboration found 1.11(8) GeV for the lightest state in the $0^{++}$channel [65]. McNeile and Michael [66] in an unquenched lattice QCD calculation focused on the mass difference (in the hope that systematics cancel) between the $1^{+-}$and the $0^{++}$mesons. Using this mass splitting it was claimed that the lightest state in the $0^{++}$ channel was consistent with the $a_{0}(980)$ state. Lang et al. reported masses for the lightest flavor nonsinglet $0^{++}$ consistent with the mass of the $a_{0}(980)$ meson, from an unquenched lattice QCD calculation using chirally improved fermions [67]. In an unquenched lattice QCD calculation with $2+1$ flavors of sea quarks, Lin et al. [68] found the lightest $a_{0}$ state to be consistent with the experimental $a_{0}(980)$.

One complication is that experimentally the $a_{0}$ decays to $\pi \eta$. In the two-flavor world, the lightest $\eta$ meson is the flavor singlet pseudoscalar meson at the $800 \mathrm{MeV}$ level. It is the mixing between the light and strange loops in a lattice calculation that drives the mixing between flavor singlet pseudoscalar states $\eta$ and $\eta^{\prime}$. Hence, the decay thresholds will be very different for the $n_{f}=2$ and $n_{f}=$ $2+1$ calculations that involve decay to a flavor singlet pseudoscalar meson. The ETM Collaboration has recently published the masses of the flavor singlet pseudoscalar meson (called $\eta_{2}$ ) on these ensembles [69] and these results will be used to estimate decay thresholds here. In Fig. 5 we plot the $a_{0}$ data and the decay thresholds.

To learn how to deal with mesons with open decays on the lattice, we need some simple test cases to validate the lattice methods. A bad example to study would be the $a_{1}(1260)$ because of its large experimental decay width of 250 to $600 \mathrm{MeV}$ [70]. The $b_{1}(1235)$ meson is good choice, because most models treat it as a $\bar{q} q$ state and its width is not too large at $142 \mathrm{MeV}$ [70]. A direct study of the decay transition $b_{1} \rightarrow \omega \pi$ has been made on the lattice with acceptable agreement [60] with the experimental decay width. To illustrate the impact of this ( $S$-wave) decay threshold on the $b_{1}$ meson, we can use the $\rho \pi$ decay threshold (because the difference between the $\rho$ and $\omega$ masses is shown to be small [63]).

In Fig. 6 we plot our results from the ETM Collaboration for the mass of the $b_{1}$ meson with the estimate of the $\omega \pi$ threshold, as a function of the square of the pion mass. The mass of the lightest state in the $b_{1}$ channel is above the decay threshold. This necessitates including the $\omega \pi$ operators with the $b_{1}$ operators in a variational analysis, which we plan to do in future work.

In Fig. 7 we plot the mass difference between the mass of the $b_{1}$ and $a_{0}$ meson as a function of the square of the pion masses from a collection of recent unquenched lattice calculations. The fact that the majority of the results show the mass of the $a_{0}$ meson to be lighter than the mass of the $b_{1}$ meson is good evidence for the lightest $a_{0}$ on the lattice corresponding to the experimental $a_{0}(980)$ state. The Kentucky group has recently stressed that the identification

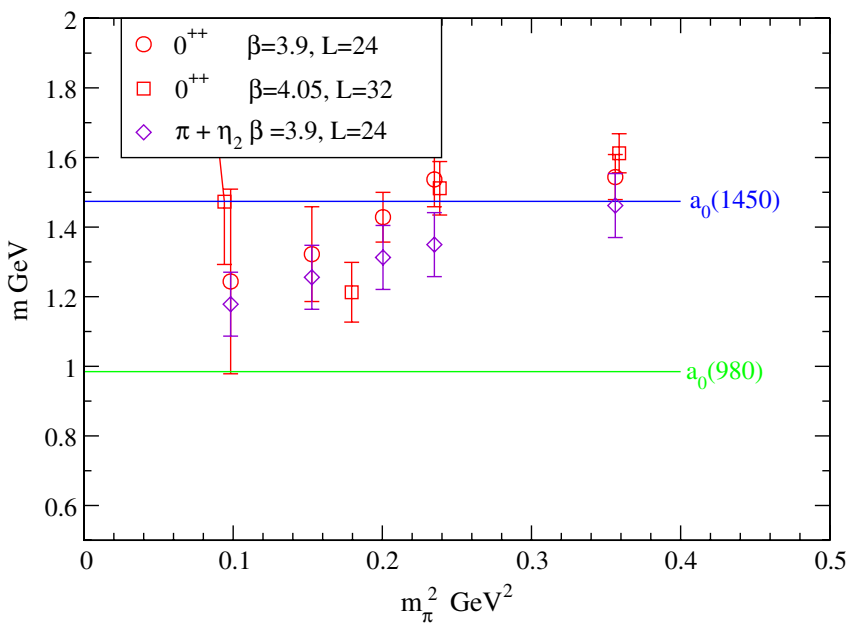

FIG. 5 (color online). Mass of lightest state in $0^{++}$channel with the $\pi \eta_{2}$ decay threshold. 


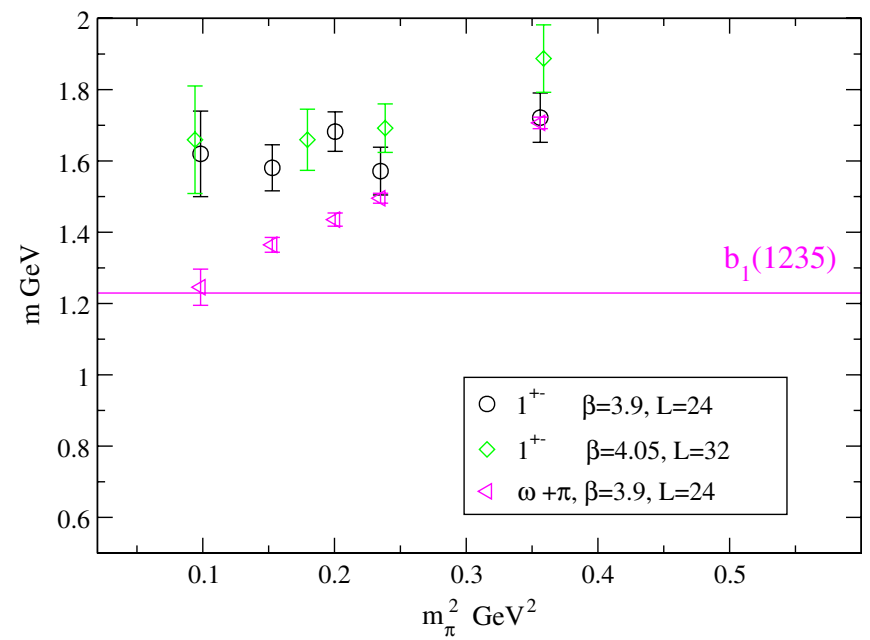

FIG. 6 (color online). Mass of $b_{1}$ state and $\pi \omega$ threshold as a function of square of pion mass.

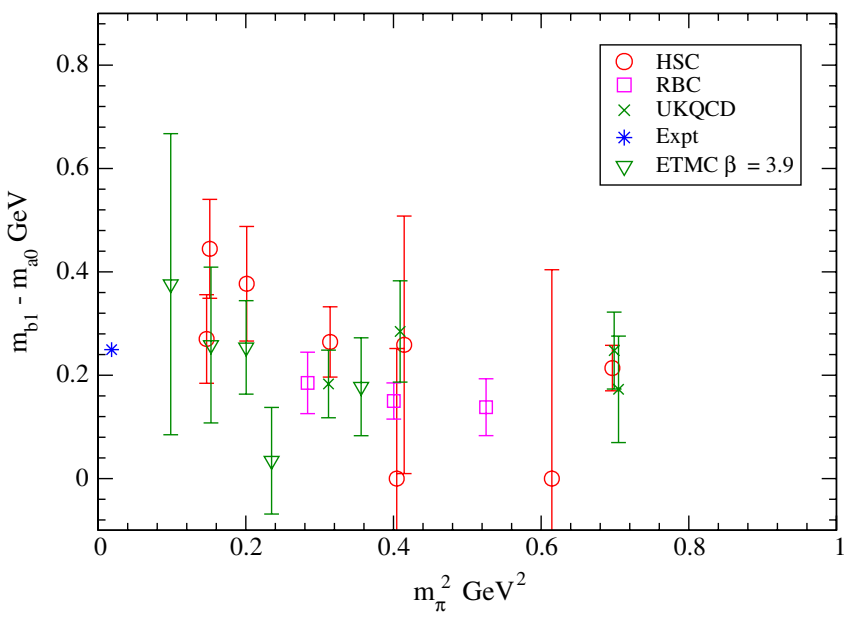

FIG. 7 (color online). Mass splitting between the $b_{1}$ and $a_{0}$ mesons. The plot includes data from the RBC Collaboration [65], Hadron Spectrum Collaboration (HSC) [68], the UKQCD Collaboration [66], and the ETMC results from this work.

of the $a_{0}(980)$ state on the lattice requires an understanding of dynamics of the strong decay [71].

\section{RESULTS FOR THE MASSES OF THE LIGHT $1^{--}$MESON}

In this section we will discuss the physical results for the mass of the vector mesons. There is much more information on effective field theory for the vector mesons, so there is more we can do with the chiral extrapolations in the mass of the light quarks. The data for the $\rho$ meson are useful for applications such as the calculation of the vacuum polarization tensor that is part of the QCD corrections to $g-2$ $[13,14]$ and the comparison of the electromagnetic form factor of the pion with the vector exchange model [54].

In Fig. 8 we plot the mass of the lightest vector meson as a function of the square of the pion mass. Our lattice data

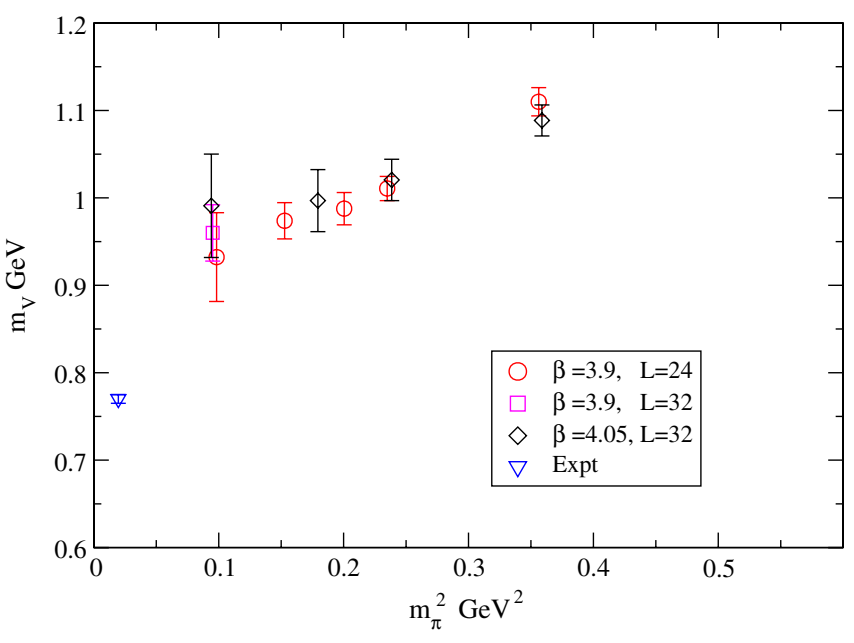

FIG. 8 (color online). The mass of the light vector meson as a function of the square of the light pseudoscalar meson.

seem high relative to the experimental mass of the $\rho$ meson. A more detailed comparison with experiment requires a discussion of the chiral extrapolations. Also the effect of $\rho$ decay needs to be accounted for. There has been a long history of attempts to deal theoretically with the effect of the $\rho$ decay on the mass of the $\rho$ meson [72-76].

In [7] the vector meson mass as a function of the square of the pion mass was plotted with data from lattice QCD calculations that used improved staggered (MILC Collaboration [22]), and domain wall fermions (RBCUKQCD [77]). There was reasonable agreement between the data from the different formalisms, although the statistical errors need to be reduced on some results (including ours).

Lattice correlators should have a signal to noise ratio which goes like $e^{-\left(m_{M}-m_{\pi}\right) t}$ for a meson of mass $m_{M}$ [78]. We have checked that our data at $\beta=3.9$ obeys this relation. So there is no fundamental problem with the increase in the statistical errors as the mass of the light quarks is reduced. On a subset of the configurations we tried a technique called color dilution to improve the signal to noise ratio for the connected $\rho$ correlators [79]. This did not reduce the statistical noise. ETMC have used an extrapolation of the partially quenched $\rho$ masses to reduce the statistical errors [80].

At $\beta=3.9$ and $\mu=0.004$, we have also estimated the mixing element between $\rho^{0}$ and $\pi^{+} \pi^{-}$from a correlator ratio using the method described in [20]. The three-point function ratio was computed using

$$
c_{3}(t)=\frac{\langle\rho(0) \mid \pi(t) \pi(t)\rangle}{\langle\rho(0) \mid \rho(t)\rangle^{1 / 2}\langle\pi(0) \pi(0) \mid \pi(t) \pi(t)\rangle^{1 / 2}}
$$

When the $\rho$-mass and $\pi \pi$ energy are degenerate, for small enough $x$ [20] (where $x=\langle\rho \mid \pi \pi\rangle$ ), this ratio can be fitted to the model in Eq. (9). 


$$
c_{3}(t) \rightarrow x t+\text { const. }
$$

The formalism required, where the $\rho$-mass and $\pi \pi$ energy are not degenerate, is discussed in [81]. The correlator ratio $c_{3}(t)$ is plotted in Fig. 9 for the decay $\rho \rightarrow \pi(k=$ $\left.\frac{2 \pi}{L}\right) \pi\left(k=-\frac{2 \pi}{L}\right)$. Since the $\rho$-mass is somewhat larger (by 0.19 in lattice units) than the lightest two-pion energy, we plot in the figure a theoretical curve which modifies Eq. (9), taking this into account, as used in Ref. [20]. This fit to the three-point function ratio gives $a x=0.060(15)$. Since on a lattice, energy is not conserved, we have evaluated the transition amplitude to a final state with sufficient momentum that its energy is more than that of the $\rho$ at rest, so strictly a zero decay width. So, to compare with experiment, it is optimum to evaluate the coupling constant. This may have some dependence on momentum in general, but it is a useful point of reference. The $g_{\rho \pi \pi}^{2}$ coupling defined via

$$
\Gamma=\frac{g_{\rho \pi \pi}^{2}}{6 \pi} \frac{k^{3}}{m_{\rho}^{2}}
$$

is found to be $g_{\rho \pi \pi}=5.2(1.3)$. The corresponding value of $g_{\rho \pi \pi}$ from the experimental value of the $\rho$ width is 6.0. So we have consistency between the lattice estimate of the coupling between $\rho$ and $\pi \pi$ and that observed.

Since we measure the strength of the transition from $\rho$ to $\pi \pi$ on the lattice (namely $x$ ), we can estimate the mass shift caused by this mixing. Then with a two-state model with energy difference $\Delta$ where

$$
\Delta=E_{2}-E_{1}=2 \sqrt{m_{\pi}^{2}+\left(\frac{2 \pi}{L}\right)^{2}}-m_{\rho}
$$

with $a \Delta=0.19$ in our case, we obtain, using [20], a shift (downwards for the $\rho$ ) of

$$
m_{\text {split }}=\sqrt{\Delta^{2} / 4+x^{2}}-\Delta / 2
$$

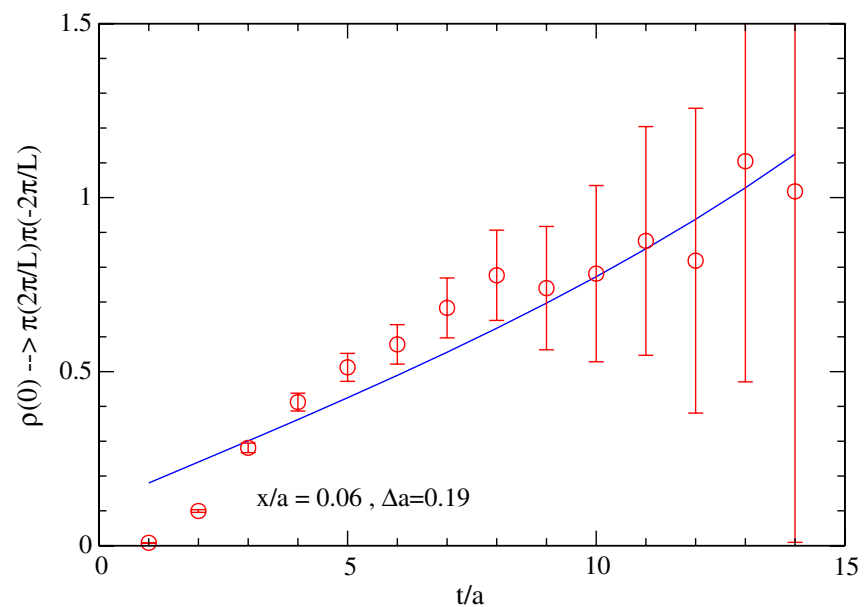

FIG. 9 (color online). The correlator $c_{3}(t)$ in Eq. (8) as a function of time.
This mixing produces a $4 \%$ downward shift in the mass of the $\rho$ for ensemble $B_{1}$ using this simplified mixing scheme. This shift is comparable to our statistical error for that state. This suggests that the mass of the vector mesons in Fig. 8 are largely unaffected by the two $\pi$ decay.

This mixing argument can be used to compare expectations between the $B_{6}$ ensemble with $L=32$ and that above with $L=24$ above. The differences will be that the energy gap will become much smaller $(\Delta=0.08)$ since the minimum momentum is reduced while the mixing contribution $\left(x^{2}\right)$ will be reduced proportionally to the spatial volume. The net effect is a rather similar estimate which is consistent with our results which show that the $\rho$ mass from the $B_{6}$ ensemble is half- $\sigma$ higher than for the $B_{1}$ ensemble.

We now discuss the chiral extrapolation of the vector masses to the physical point. For the case of an effective field theory for vector mesons, the issues in writing down an effective field theory are less clear than for pions. A fully relativistic Lagrangian can be used for the vector fields or a heavy meson effective theory (HMET) $[16,82]$. The connection between the different effective theories is discussed in [16,83].

The most basic effective field theory for the light vector meson predicts that the mass of the vector meson depends on the mass of the pion via $[16,82]$ :

$$
M_{\rho}=M_{\rho}^{0}+c_{1} M_{\pi}^{2}+c_{2} M_{\pi}^{3} .
$$

The pions involved in $\rho$ decay are not soft so $\rho \rightarrow \pi \pi$ can not be studied using chiral perturbation theory with power counting [16,82]. However, Bijnens et al. [16] successfully fitted the masses of the light vector mesons $\rho$ to $\phi$, including electromagnetic effects, using HMET but not including the dynamics of the $\rho \rightarrow \pi \pi$ decay.

The $\rho$ decay will affect the chiral extrapolation model used to extrapolate the mass of the $\rho$ meson. The Adelaide group has studied different regulators [74-76] for the effective field theory of $\rho$ decay. This produced additional mass dependence at very light pion masses.

Models for the effect of $\pi \omega$ and $\pi \pi$ contributions to the mass of the $\rho$ meson have direct implications for the mass of the $\omega$ meson (which has $\pi \rho$ contributions). Hence lattice results for the quark dependence of the mass splitting of the $\omega$ to $\rho$ mesons [63] allow further constraints to the study of individual terms.

Bruns and Meißner [84] have published a chiral extrapolation formula for the mass of the $\rho$ meson. The derivation used a modified $\overline{M S}$ regulator and a power counting scheme.

$$
M_{\rho}=M_{\rho}^{0}+c_{1} M_{\pi}^{2}+c_{2} M_{\pi}^{3}+c_{3} M_{\pi}^{4} \ln \left(\frac{M_{\pi}^{2}}{M_{\rho}^{2}}\right) .
$$

The term with the $c_{3}$ coefficient is due to the self-energy (in the infinite volume limit). Bruns and Meißner [84] recommend that the size of the $c_{i}$ coefficients obtained from the fits to the lattice calculations be checked against con- 
TABLE III. The $\rho$ mass from chiral extrapolation from different fit models at $\beta=3.9$.

\begin{tabular}{llclcccc}
\hline \hline Equation & Model & $m_{\rho} \mathrm{GeV}$ & $m_{\phi} \mathrm{GeV}$ & $M_{\rho}^{0} \mathrm{GeV}$ & $c_{1} \mathrm{GeV}^{-1}$ & $c_{2}(\mathrm{GeV})^{-2}$ & $c_{3}(\mathrm{GeV})^{-3}$ \\
\hline$(13)$ & linear & $0.90(4)$ & $1.13(8)$ & $0.89(5)$ & $0.49(26)$ & $\ldots$ & $\ldots$ \\
$(14)$ & Bruns and Meißner & $0.90(5)$ & $1.07(10)$ & $0.89(6)$ & $3.5(4.7)$ & $-0.09(81)$ & $-0.82(41)$ \\
\hline \hline
\end{tabular}

straints from low-energy effective constants. However they only quote, as reasonable, the constraints that $\left|c_{i}\right|<3$. The Adelaide group [74] claimed to know the sign and magnitude of the $c_{2}$ coefficient $\left(c_{2} \sim-1.70 \mathrm{GeV}^{-2}\right)$, but Bruns and Meißner [84] claim their bounds are more general.

Using one-loop chiral perturbation theory and a technique called the inverse amplitude method, Hanhart et al. $[85,86]$ estimate $c_{1}=0.90 \pm 0.11 \pm 0.13 \mathrm{GeV}^{-1} M_{\rho}^{0}=$ $0.735 \pm 0.0017 \mathrm{GeV}$.

Bruns and Meißner, [84] from an analysis of an old lattice QCD calculation by the CP-PACS Collaboration [87], found that the curvature from the nonanalytic terms can produce either an increase or decrease in the vector mass over a simple linear fit. CP-PACS used the string tension $(440 \mathrm{MeV})$ to set the lattice spacing [87]; this corresponds to $r_{0} \sim 0.54 \mathrm{fm}$, roughly $10 \%$ higher than the preferred $r_{0}$ from the pion decay constant. If there is any ambiguity in the lattice spacing, then this can hide the curvature from the nonanalytic terms.

Unfortunately the size of errors on the $\rho$ data and the number of points does not allow us to include the $c_{2}$ and $c_{3}$ coefficients as free parameters. To get some idea of the effect of these terms we use the augmented $\chi^{2}$ method $[88,89]$ where the physics constraints from Bruns and Meißner [84] can be built into the fit with Bayesian techniques. The augmented $\chi^{2}$ is used to constrain $c_{2}$ and $c_{3}$.

$$
\chi_{\text {aug }}^{2}=\chi^{2}+\sum_{j=2}^{3} \frac{\left(c_{i}-0\right)^{2}}{3^{2}} .
$$

Schindler and Phillips have recently discussed using an augmented $\chi^{2}$ to using information from effective theories in chiral extrapolations of lattice data. We use the bootstrap method to estimate the errors. In principle given the probability distribution, the errors on the parameters can be obtained by integrating the Monte Carlo integrals [89,90]. Chen et al. checked [91] that consistent errors were obtained from a bootstrap analysis and from an error analysis based on the augmented $\chi^{2}$ being a quadratic function of the fit parameters around the minimum.

We also investigated an approach developed by the Adelaide [74] group. The Adelaide method uses a dipole regulator, rather than the $\overline{M S}$ scheme, to regulate the effective field theory corrections to the $\rho$ mass [74]. The extrapolation model for the mass of the $\rho$ meson is

$$
M_{\rho}=M_{\rho}^{0}+c_{1} M_{\pi}^{2}+\frac{\Sigma_{\pi \omega}\left(\Lambda_{\pi \omega}, M_{\pi}\right)+\Sigma_{\pi \pi}\left(\Lambda_{\pi \pi}, M_{\pi}\right)}{2\left(M_{\rho}^{0}+c_{1} M_{\pi}^{2}\right)}
$$

where $\Sigma_{\pi \omega}$ and $\Sigma_{\pi \pi}$ are the self-energies from the $\pi \pi$ and $\pi \omega$ states. The fit parameters in Eq. (16) are $M_{\rho}^{0}, c_{1}$ and $\Lambda_{\pi \omega}$. The parameter $\Lambda_{\pi \pi}$ is related to $\Lambda_{\pi \omega}$. The $\rho$ selfenergy contribution $\Sigma_{\pi \pi}$ contains a cut at $m_{\rho}=2 m_{\pi}$ for the decay $\rho \rightarrow \pi \pi$. For the continuum integral we used the principle value of the integral when the decay is open. We found that our data was too noisy to get stable fits from this method. We were also unable to resolve the quadratic $c_{2}$ term in Eq. (13), because the error bars were too large. The original study [74] of Eq. (16) used $\rho$ masses from lattice QCD with $1 \%$ errors at heavier quark masses [74].

The summary of the final results is in Table III. We use the pion mass of $135 \mathrm{MeV}$, because we do not include any electromagnetism in the lattice calculation. We also extrapolate our results to mass of the notional strange-strange pseudoscalar meson $(696 \mathrm{MeV})$. We call this the unitary $\phi$ analysis. Note that a better approach to the $\phi$ meson within an $n_{f}=2$ formalism would be to treat the strange quark as a (partially quenched) valence quark with a sea of light quarks.

In Fig. 10 we plot the linear fit and the extrapolation model in Eqs. (14).

The lattice data for the vector mesons seem to prefer a smaller lattice spacing than the scales obtained from the pion decay constant [47] and the nucleon mass [49]. This is probably because we are missing some of the effect from

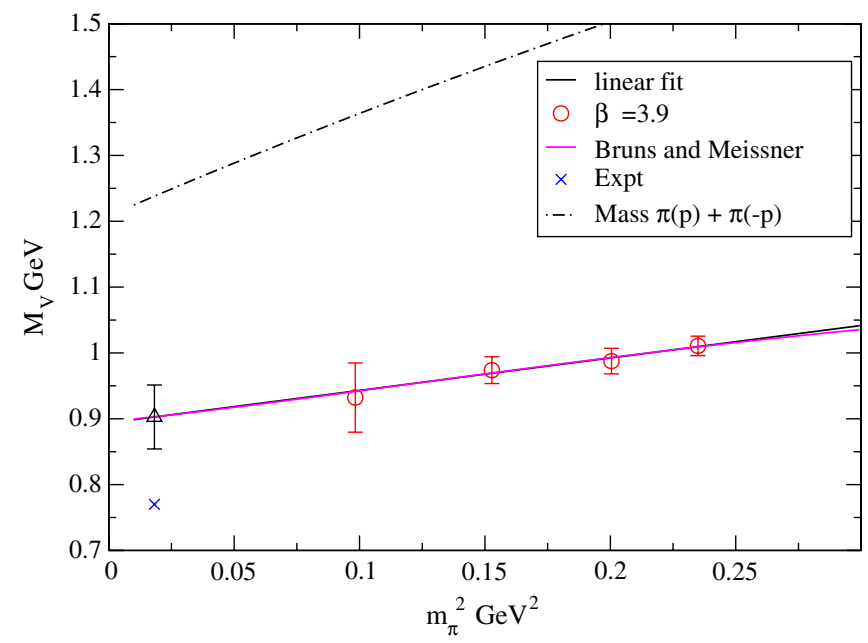

FIG. 10 (color online). Fit to the mass of the vector meson using a linear fit in the square of the pion mass and Eq. (14) at $\beta=3$.9. Also included in the plot is the first decay threshold to $\pi \pi$ for $L=24$. 
the $\rho$ decay and possibly also from the dynamical strange quark.

\section{THE DECAY CONSTANTS OF THE $\rho$ AND $\phi$ MESONS}

We first introduce the leptonic decay constant of the vector mesons, such as the $\rho$ or $\phi$, in the continuum [92]. The decay constant of the vector meson $V$ is defined [93] via

$$
\left\langle 0\left|V_{\mu}\right| V\right\rangle=m_{\rho} f_{V} \epsilon_{\mu}
$$

where the vector current is defined via

$$
V_{\mu}(x)=\bar{\psi}(x) \gamma_{\mu} \psi(x) .
$$

There are other possible (slightly different) definitions of the decay constant of the $\rho$ definitions, for example, as used by Lewis and Woloshyn [92].

The decay constants of the $\rho$ and $\phi$ mesons can be extracted from $\tau$ decay and $e^{+} e^{-}$annihilation (see [94,95] for a discussion).

$$
\begin{gathered}
f_{\rho^{+}}^{\text {expt }} \sim 208 \mathrm{MeV} \\
f_{\rho^{0}}^{\text {expt }} \sim 216(5) \mathrm{MeV} \\
f_{\phi}^{\text {expt }} \sim 233 \mathrm{MeV} .
\end{gathered}
$$

The difference between the experimental values of the $f_{\rho^{+}}^{\text {expt }}$ and $f_{\rho^{0}}^{\text {expt }}$ is probably due to the problems of extracting the parameters of the $\rho$ meson from experimental data, rather than electromagnetic effects that are important for light pseudoscalar mesons [96].

The transverse decay constant $\left(f_{V}^{T}(\mu)\right)$ of the $V$ meson is defined by

$$
\left\langle 0\left|\bar{\psi} \sigma_{\mu \nu} \psi\right| V\right\rangle=i f_{V}^{T}(\mu)\left(p_{\mu} \epsilon_{\nu}-p_{\nu} \epsilon_{\mu}\right)
$$

where $\sigma_{\mu \nu}=i / 2\left[\gamma_{\mu}, \gamma_{\nu}\right]$. It is convenient to introduce the tensor current $T_{\nu \mu}=\bar{\psi} \sigma_{\mu \nu} \psi$. In the lattice calculations we do not include any momentum.

There is no experimental result for the tensor decay constant $f_{V}^{T}(\mu)$ for the $\rho$ or $\phi$ mesons. However, light cone sum rules require the transverse decay constant of the $\rho$ meson $[97,98]$ for the extraction of $\frac{\left|V_{t t}\right|}{\left|V_{t s}\right|}$ from the $B \rightarrow$ $\rho \gamma$ and $B \rightarrow K^{\star} \gamma$ decays. The transverse decay constant of the $\rho$ meson is also used in the analysis of other $B$ decays [12]. There have been previous lattice QCD calculations of the transverse decay constants of the $\rho$ meson [94,99-101].

There needs to be a way to estimate the effect of the strong decay of the $\rho$ meson to two $\pi$ on the decay constants, in the same way we tried for the $\rho$ mass in Sec. V. A simple test is look at the $f_{V}$ decay constant for the $\rho$ and $\phi$ mesons as these give us an estimate of our accuracy. The majority of older lattice QCD calculations concentrated on the ratio of $f_{V}^{T}$ to $f_{V}$.

There are various correlators that can be used to extract the $f_{V}$ and $f_{V}^{T}$ decay constants, for example, the correlators in Eqs. (23)-(25). Our results are based on factorizing fits to a basis of 4 by 4 smearing functions that include the local operators as matrix elements in the smearing matrix, so the operators in Eqs. (23)-(25) are included.

$$
\begin{aligned}
\sum_{x} \sum_{\mu=1}^{3}\left\langle V_{\mu}\left(x, t_{x}\right) V_{\mu}(0,0)^{\dagger}\right\rangle & \rightarrow \frac{3 m_{V} f_{V}^{2} e^{-m_{V} t_{x}}}{2} \\
\sum_{x} \sum_{\mu=1}^{3}\left\langle T_{\mu 0}\left(x, t_{x}\right) V_{\mu}(0,0)^{\dagger}\right\rangle & \rightarrow \frac{3 f_{V} f_{V}^{T} m_{V} e^{-m_{V} t_{x}}}{2} \\
\sum_{x} \sum_{\mu=1}^{3}\left\langle T_{\mu 0}\left(x, t_{x}\right) T_{\mu 0}(0,0)^{\dagger}\right\rangle & \rightarrow \frac{3 m_{V}\left(f_{V}^{T}\right)^{2} e^{-m_{V} t_{x}}}{2} .
\end{aligned}
$$

The local vector $V_{\mu}$ and $T_{\mu \nu}$ tensor currents need to be renormalized. This involves some discussion of the twisted mass formalism. We do all our fits in the twisted bases, however the identification of states is done in the physical basis [48]. Assuming that the calculations are done at maximal twist (see Eq. (6)), we have

$$
\begin{gathered}
\left\langle i\left|V_{\mu}^{3}\right| j\right\rangle_{\text {cont }}=Z_{V}\left\langle i\left|V_{\mu}^{3}\right| j\right\rangle_{\text {twisted lattice }} \\
\left\langle i\left|V_{\mu}^{\alpha}\right| j\right\rangle_{\text {cont }}=Z_{A} \epsilon^{3 \alpha \beta}\left\langle i\left|A_{\mu}^{\beta}\right| j\right\rangle_{\text {twisted lattice }} \\
\left\langle i\left|T_{\nu \mu}^{\alpha}\right| j\right\rangle_{\text {cont }}=Z_{T}\left\langle i\left|T_{\nu \mu}^{\alpha}\right| j\right\rangle_{\text {twisted lattice }}
\end{gathered}
$$

where $\alpha$ takes the values of 1 or 2 . Given that we found that the disconnected graphs for vector mesons were negligible (in Sec. IV), then the connected charged and neutral vector mesons give us a separate estimate of the decay constants that use different renormalization constants. This is a useful test of the renormalization and cutoff effects.

The relevant renormalization factors $Z_{V}, Z_{T}$, and $Z_{A}$, have been computed [102,103] using the RomeSouthampton nonperturbative method [104]. The $Z_{V}$ factor has also been computed using the conserved vector current [48]. It was found that the conserved vector current produced a more accurate estimate of $Z_{V}$ than the RomeSouthampton method, so we use the result from the conserved current in this analysis. In this paper we use the $Z_{A}$ and $Z_{T}$ values calculated through the "p2-window" method without the use of the subtraction of $O\left(a^{2} g^{2}\right)$ terms. In Table IV we summarize the renormalization factors used in this calculation [102,103].

The value of the tensor current depends on the scale. The tensor current at $\mu_{a}^{2}$ is obtained from that at another scale $\left(\mu_{b}^{2}\right)$ by using the renormalization group equation.

$$
Z_{T}\left(\mu_{a}^{2}\right)=\frac{C\left(\mu_{a}^{2}\right)}{C\left(\mu_{b}^{2}\right)} Z_{T}\left(\mu_{b}^{2}\right)
$$


TABLE IV. Summary of the nonperturbative renormalization factors used in this calculation. The $C(\mu)$ function is the solution, in Eq. (30), of the RG equation for the tensor current.

\begin{tabular}{lcccc}
\hline \hline$\beta$ & $Z_{A}$ & $Z_{T}\left(\mu=\frac{1}{a}\right)$ & $Z_{V}$ & $\frac{C(2 \mathrm{GeV})}{C\left(\mu=\frac{1}{-1}\right)}$ \\
\hline 3.9 & $0.771(4)$ & $0.769(4)$ & $0.6104(02)$ & 1.01 \\
4.05 & $0.785(6)$ & $0.787(7)$ & $0.6451(02)$ & 1.03 \\
\hline \hline
\end{tabular}

$$
\begin{aligned}
C\left(\mu^{2}\right)= & \left(\frac{\alpha_{s}(\mu)}{\pi}\right)^{\gamma_{0}}\left[1+\left(\frac{\alpha_{s}(\mu)}{\pi}\right)\left(\bar{\gamma}_{1}-\bar{\beta}_{1} \bar{\gamma}_{0}\right)\right. \\
& +\frac{1}{2}\left(\frac{\alpha_{s}(\mu)}{\pi}\right)^{2}\left[\left(\bar{\gamma}_{1}-\bar{\beta}_{1} \bar{\gamma}_{0}\right)^{2}+\bar{\gamma}_{2}+\bar{\beta}_{1}^{2} \bar{\gamma}_{0}\right. \\
& \left.\left.-\bar{\beta}_{1} \bar{\gamma}_{1}-\bar{\beta}_{2} \bar{\gamma}_{0}\right]\right]
\end{aligned}
$$

with

$$
\begin{gathered}
\bar{\gamma}_{i}=\frac{\gamma_{i}}{\beta_{0}}, \quad \bar{\beta}_{i}=\frac{\beta_{i}}{\beta_{0}} \\
\beta_{0}=\frac{1}{4}\left(11-\frac{2}{3} n_{f}\right) \quad \beta_{1}=\frac{1}{16}\left(102-\frac{38}{3} n_{f}\right) \\
\beta_{2}=\frac{1}{64}\left(\frac{2857}{2}-\frac{5033 n_{f}}{18}+\frac{325 n_{f}^{2}}{54}\right) .
\end{gathered}
$$

The anomalous dimension for the tensor current has been computed by Gracey $[105,106]$ to three loops in the $R I^{\prime}$ and the $\overline{M S}$ schemes.

$\gamma_{0}=\frac{1}{3} \quad \gamma_{1}=\frac{543-26 n_{f}}{216}$
$\gamma_{2}=-\left(\frac{36 n_{f}^{2}+1440 \zeta_{3} n_{f}+5240 n_{f}+2784 \zeta(3)-52555}{5184}\right)$

where the value of the standard constant is $\zeta_{3}=1.20206$.

For the coupling we used the RunDec package [107] to compute the coupling from $\Lambda_{\mathrm{QCD}}$ using four-loop evolution $[108,109]$. There has not been a calculation of the strong coupling using information from these configurations. We used the value of $\Lambda_{\mathrm{QCD}}=261$ (17)(26) MeV from QCDSF [110]. The QCDSF value is consistent with that from ALPHA [111], that also used $n_{f}=2$ QCD.

The results for the leptonic decay constant are reported in Table $\mathrm{V}$ and the results for the transverse $\rho$ decay constant are in Table VI. The decay constants from the neutral and charged vector mesons agree within the errors. We now only consider the decay constants of charged vector mesons. In Fig. 11 we plot the decay constant of the vector meson as a function of the square of the pion mass. There is reasonable scaling between the decay constants at $\beta=3.9$ and $\beta=4.05$. The data with larger masses also disagree with the value of the decay constant of the $\phi$ meson. The $\phi$ has a small decay width
TABLE V. Summary of the leptonic decay constant of the vector meson for the different ensembles from this calculation.

\begin{tabular}{lllcc}
\hline \hline & \multicolumn{2}{c}{ Charged } & \multicolumn{2}{c}{ Neutral } \\
Ensemble & $a f_{V} / Z_{A}$ & $f_{V} \mathrm{MeV}$ & $f_{V} \mathrm{MeV}$ & $a f_{V} / Z_{V}$ \\
\hline$B_{1}$ & $0.13(1)$ & $234(18)$ & $252(13)$ & $0.179(9)$ \\
$B_{2}$ & $0.148(4)$ & $264(7)$ & $283(18)$ & $0.20(1)$ \\
$B_{3}$ & $0.149(4)$ & $265(7)$ & $274(14)$ & $0.19(1)$ \\
$B_{4}$ & $0.151(4)$ & $269(7)$ & $\ldots$ & $\ldots$ \\
$B_{5}$ & $0.162(4)$ & $289(11)$ & $\ldots$ & $\ldots$ \\
$B_{6}$ & $0.151(7)$ & $269(12)$ & $275(19)$ & $0.19(1)$ \\
$C_{1}$ & $0.119(10)$ & $277(24)$ & $306(22)$ & $0.16(1)$ \\
$C_{2}$ & $0.117(6)$ & $272(14)$ & $291(14)$ & $0.152(7)$ \\
$C_{3}$ & $0.117(4)$ & $272(10)$ & $\cdots$ & $\cdots$ \\
$C_{4}$ & $0.121(3)$ & $281(9)$ & $\ldots$ & $\ldots$ \\
\hline \hline
\end{tabular}

(4.26(4) MeV), so we might expect to be able get the properties of this meson correctly. However, the $\phi$ is considered to be mostly $\bar{s} \gamma_{\mu} s$, so our neglect of the dynamics of the strange quark could be important.

It has been found that chiral perturbation theory is required to extrapolate the decay constants of the light pseudoscalar mesons to their values at the physical quark masses [2,3]. As discussed in Sec. V the application of effective Lagrangian techniques to study the $\rho$ meson is problematic because of the large mass of the $\rho$ meson relative to the chiral scale [84]. There are expressions for quark mass dependence of the vector meson decay constants in [112]. The corrections due to loops start at $m_{q} \log m_{q}$ and $m_{q}^{3 / 2}$. Given the size of the statistical errors on the decay constants we did not try to include any chiral corrections in the chiral extrapolations. A simple fit, linear in the square of the pion mass, of the $\beta=3.9$ data gives $f_{\rho}^{\text {phys }}=239(18) \mathrm{MeV}$ and $f_{\phi}^{\text {phys }}=308(29) \mathrm{MeV}$.

At the moment there are no results for the mass dependence of the transverse leptonic decay constants from effective field theory, however the formalism for tensor currents has started to be developed $[113,114]$. It will be interesting to see the predictions for the mass dependence

TABLE VI. Summary of the transverse decay constant $\left(f_{V}^{T}(\mu)\right)$ of the vector meson. The scale is $\mu=2 \mathrm{GeV}$.

\begin{tabular}{lccc}
\hline \hline Ensemble & $a f_{V}^{T} / Z_{T}$ & $f_{V}^{T}(2 \mathrm{GeV}) \mathrm{MeV}$ & $\frac{f_{V}^{T}(2 \mathrm{GeV})}{f_{V}}$ \\
\hline$B_{1}$ & $0.108(8)$ & $194(15)$ & $0.83(4)$ \\
$B_{2}$ & $0.109(3)$ & $195(5)$ & $0.74(2)$ \\
$B_{3}$ & $0.111(2)$ & $198(6)$ & $0.75(1)$ \\
$B_{4}$ & $0.113(3)$ & $203(6)$ & $0.75(1)$ \\
$B_{5}$ & $0.128(5)$ & $218(8)$ & 0.78() \\
$B_{6}$ & $0.109(5)$ & $196(9)$ & $0.73(2)$ \\
$C_{1}$ & $0.089(7)$ & $214(18)$ & $0.77(5)$ \\
$C_{2}$ & $0.081(4)$ & $193(9)$ & $0.71(2)$ \\
$C_{3}$ & $0.089(3)$ & $214(8)$ & $0.79(2)$ \\
$C_{4}$ & $0.090(3)$ & $215(7)$ & $0.76(1)$ \\
\hline \hline
\end{tabular}




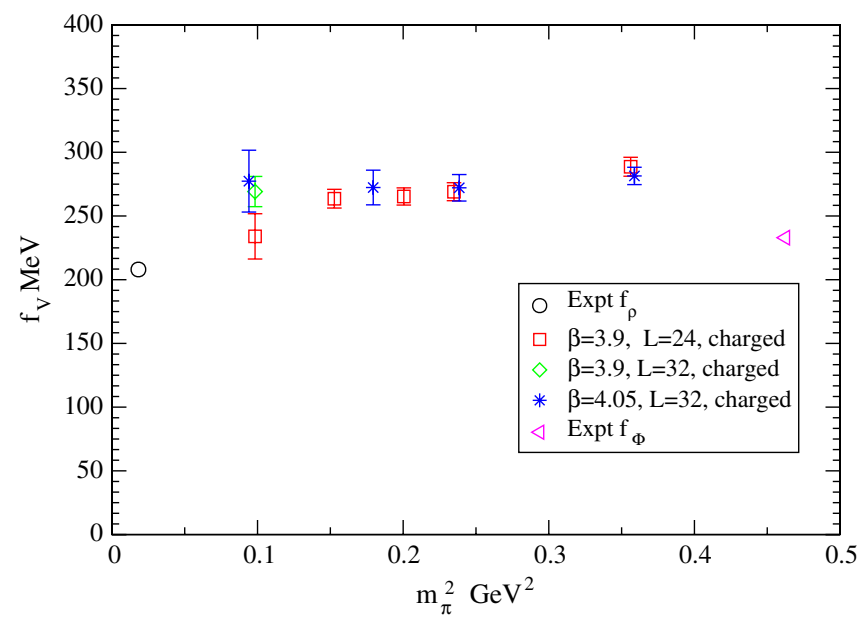

FIG. 11 (color online). The leptonic decay constant of the vector meson (as defined in Eq. (17)) is plotted as a function of the square of the pion mass. The experimental points for the $\rho$ and $\phi$ are also included.

of the ratio of the transverse to leptonic decay constant from effective field theory, because this will test whether a chiral extrapolation of the ratio of the leptonic to transverse decay constant results in a cancellation of systematic errors as is hoped.

There has not been a definitive unquenched calculation of the leptonic decay constant of the $\rho$ meson, although there have been many attempts. Lewis and Woloshyn came within $1 \%$ of the experimental result for the $f_{\rho}$ in a quenched QCD calculation using the D234 improved action [92]. Lewis and Woloshyn summarize older quenched calculations [92]. SESAM reported leptonic decay constants for vector mesons that agreed with experiment at the $20 \%$ level from an unquenched lattice QCD calculation with Wilson fermions [115]. CP-PACS [93] from unquenched calculations with the tadpole improved clover action found that they could not do a reliable continuum extrapolation of $f_{\rho}$. CP-PACS [93] found the nonperturbative and perturbative renormalization factors to be very different. QCDSF obtained $f_{\rho}=256(9) \mathrm{MeV}$ from an unquenched calculation with clover fermions [100]. Hashimoto and Izubuchi [65] obtained $f_{\rho}=$ $210(15) \mathrm{MeV}$ from a $n_{f}=2$ unquenched calculations that use domain wall fermions. However this calculation also found that $r_{0}^{\text {phys }}=0.549(9)$ fm from the mass of the $\rho$ meson, so we expect that this is the reason for obtaining a number close to the physical point.

In Fig. 12 we plot the transverse decay constant of the vector meson as a function of the pion mass squared in physical units. The ratio of transverse to leptonic decay constant is plotted in Fig. 13.

A collection of results for the transverse decay constants are presented in Table VII. We also present results from using the ratio of tensor to vector correlators in the bootstrap analysis, that we call the "ratio method." In [80] the

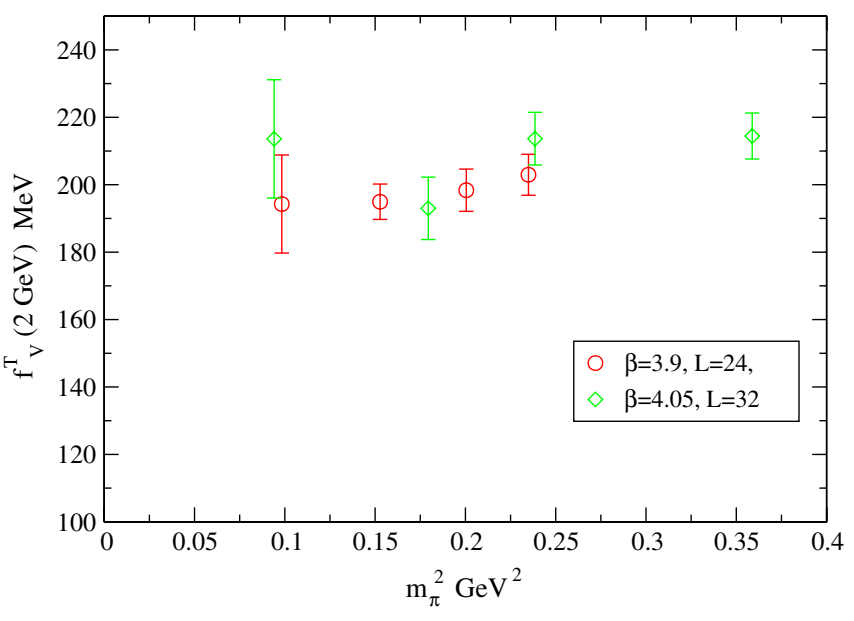

FIG. 12 (color online). The transverse decay constant of the vector meson is plotted as a function of the square of the pion mass.

ETM Collaboration presents results for $\frac{f_{K^{\star}}^{T}}{f_{K^{\star}}}$ in a partially quenched analysis on the same configurations. We see that our result for $f_{\rho}^{T}(2 \mathrm{GeV})$ is approximately $30 \mathrm{MeV}$ higher than most previous results. The RBC-UKQCD Collaboration also reports a result for the transverse decay constant of the $K^{\star}$ meson. Only QCDSF [100] compute $f_{\rho}^{T}$ on its own; all the others compute $\frac{f_{\rho}^{T}}{f_{\rho}}$ and then multiply by experiment value for $f_{\rho}$.

From what we call the unitary $\phi$ analysis we obtain $f_{\phi}^{T}=170(14) \mathrm{MeV}$ from the ratio method and $f_{\phi}^{T}=$ 222(26) MeV from the direct method, both at the scale of $2 \mathrm{GeV}$. These can be compared with the results in Table VII.

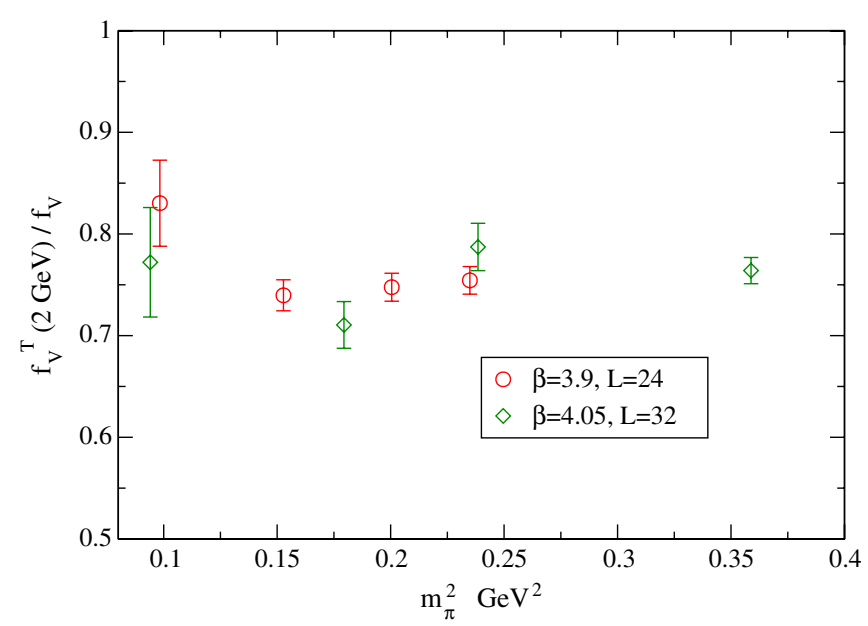

FIG. 13 (color online). The ratio of transverse to leptonic decay constant of the $\rho$ meson as a function of the square of the pion mass. The transverse decay constant is at the scale of $2 \mathrm{GeV}$. 
TABLE VII. Summary of results for transverse decay constants of the $\rho$ and $\phi$ meson. We only include the result from the finest lattice of Braun et al. [99].

\begin{tabular}{|c|c|c|c|c|}
\hline Group & Method & $f_{\rho}^{T}(2 \mathrm{GeV})$ & $f_{\phi}^{T}(2 \mathrm{GeV})$ & $\frac{f_{\rho}^{T}}{f_{\rho}}$ \\
\hline Ball et al. [116-118] & sum rule & $155(10)$ & $208(15)$ & $0.74(3)$ \\
\hline Becirevic et al. [119] & quenched lattice & $150(5)$ & $177(2)$ & $0.72(2)_{0}^{+2}$ \\
\hline Braun et al. [99] & quenched lattice & $154(5)$ & $182(2)$ & $0.74(1)$ \\
\hline QCDSF [120] & quenched lattice & $149(9)$ & $\cdots$ & $\ldots$ \\
\hline QCDSF [100] & unquenched lattice & $168(3)$ & $\cdots$ & $\cdots$ \\
\hline RBC-UKQCD [101] & unquenched lattice & $143(6)$ & $175(2)$ & $0.69(3)$ \\
\hline This work & unquenched lattice & $184(15)$ & $\ldots$ & $\ldots$ \\
\hline This work (ratio method) & unquenched lattice & $159(8)$ & $\cdots$ & $0.76(4)$ \\
\hline
\end{tabular}

Cata and Mateu [121] (see also [122]) have argued that in the large $N_{c}$ limit that $\frac{f_{\rho}^{T}}{f_{\rho}}=\frac{1}{\sqrt{2}}$. Their result is consistent with the lattice results in Table VII for both the quenched and unquenched results. There is some ambiguity in the large $N_{c}$ result, because it does not depend on the renormalization scale as it should. There are also predictions for the tensor decay constants of the excited vector mesons from large $N_{c}$ [121], that in principle could be measured in future lattice QCD calculations that use modern variational techniques [123].

\section{TESTING THE MASS DEPENDENCE OF THE KSRF RELATIONS}

In this paper we have discussed the mass of the $\rho$ meson, the lepton decay constant $f_{\rho}$, and the coupling $g_{\rho \pi \pi}$ for $\rho$ decay to $\pi \pi$. Perhaps surprisingly there are postulated connections between the three constants, that are called the KSRF relationships [124,125]. The original derivation of the KSRF relations used the application of the PCAC relation to $\rho$ decay $[124,125]$. However, the KSRF relations are also predictions of some effective field theories of mesons (see Birse for a review [126]), such as those with

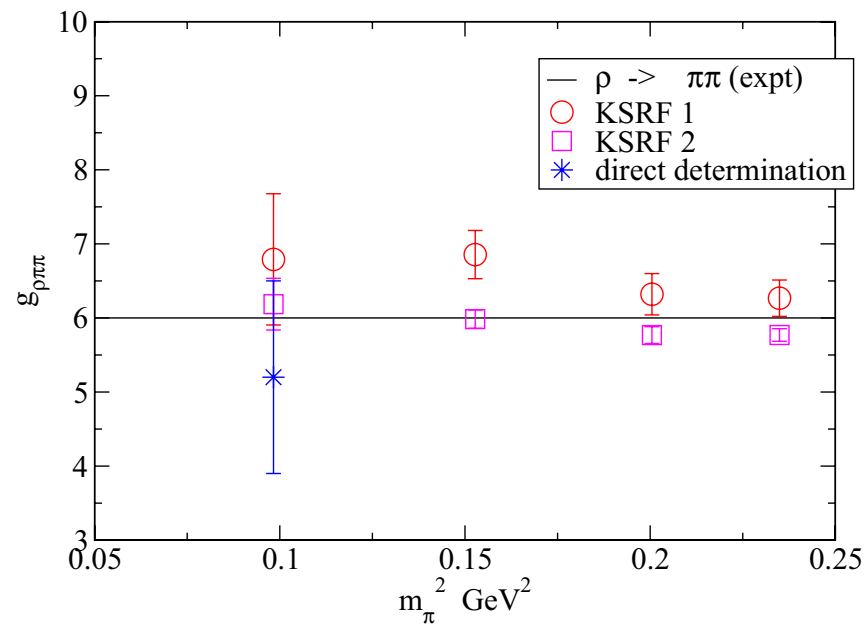

FIG. 14 (color online). Comparing the $g_{\rho \pi \pi}$ coupling from the two KSRF relations with experiment and the direct determination described in Sec. V. "hidden symmetry" [127] and the vector realization of chiral symmetry [128].

Equations (34) and (35) are known as the KSRF1 and KSRF2 relationships [127], respectively.

$$
\begin{gathered}
f_{\rho} \frac{m_{\rho}}{\sqrt{2}}=f_{\pi}^{2} g_{\rho \pi \pi} \\
m_{\rho}^{2}=f_{\pi}^{2} g_{\rho \pi \pi}^{2} .
\end{gathered}
$$

Collectively, KSRF1 and KSRF2 are known as the KSRF relations [127], named after the four authors: Kawarabayashi-Suzuki-Riazuddin-Fayyazuddin. We are using the convention where the physical pion decay constant is $f_{\pi}=130.7 \mathrm{MeV}$. In the effective field theory written down by Georgi [128], there is an additional 2 on the right-hand side of Eq. (35) that makes his model not agree with experiment very well. The KSRF relations can also be analyzed using AdS/CFT [129,130].

In Fig. 14 we plot $g_{\rho \pi \pi}$ from Eqs. (34) and (35) for the $\beta=3.9$ data, as a function of the square of the pseudoscalar meson. Within the size of the error bars, the value of $g_{\rho \pi \pi}$ is relatively independent of the pseudoscalar mass. This will be a useful test for hadronic effective field theories that include the $\rho$ meson.

Some of the work on the KSRF relation in effective field theory is used as a qualitative guide to building technicolor models of electroweak symmetry breaking $[127,128]$.

\section{CONCLUSIONS}

The first publication from the ETM Collaboration showed impressive agreement between the predictions of chiral perturbation theory and the lattice results [47]. In this paper we have found that getting agreement between the lattice results and the experimental data for the $\rho, b_{1}$, $a_{0}$ mesons is much harder. The statistical errors on the masses and couplings are too large to look for subtle effects in the chiral extrapolation models. More work, using the variational basis for the vector, the $b_{1}$ and the $a_{0}$ states and more statistics will be needed to eventually test the various chiral extrapolations.

We have started to explore using various tools, such as the Adelaide [74-76] method, computation of decay 
widths, and looking for avoided level crossings, to study resonance mesons on the lattice. Eventually, the issue of dealing with resonances in lattice QCD will use Lüscher's technique [26] and variants of [131]. These methods are computationally intensive, so more pragmatic approaches to studying strong decays on the lattice are still important at this time. Lüscher's technique for resonances was recently applied to the $\rho$ meson by the CP-PACS Collaboration [21].

\section{ACKNOWLEDGMENTS}

We thank Jozef Dudek for discussions about chiral extrapolations and open decays. We thank all members of ETMC for a very fruitful collaboration and, in particular, Giancarlo Rossi, P. Dimopoulos, Xu Feng, G. Herdoiza and S. Simula for useful comments on the paper. We acknowledge the use of the NW grid for part of this analysis.
[1] K. Jansen, arXiv:0810.5634.

[2] H. Leutwyler, arXiv:0808.2825.

[3] S. Necco, Proc. Sci., LAT2007 (2007) 021 [arXiv:0710.2444].

[4] C. A. Meyer, AIP Conf. Proc. 870, 390 (2006).

[5] M. F. M. Lutz, B. Pire, O. Scholten, and R. Timmermans (PANDA), arXiv:0903.3905.

[6] S. Prelovsek, arXiv:0804.2549.

[7] C. McNeile, Proc. Sci., LAT2007 (2007) 019 [arXiv:0710.0985].

[8] C. McNeile, arXiv:0710.2470.

[9] K.-F. Liu, arXiv:0805.3364.

[10] C. B. Lang, Prog. Part. Nucl. Phys. 61, 35 (2008).

[11] C. Gattringer, arXiv:0711.0622.

[12] P. Ball and R. Zwicky, Phys. Rev. D 71, 014029 (2005).

[13] C. Aubin and T. Blum, Phys. Rev. D 75, 114502 (2007).

[14] D. B. Renner and X. Feng, arXiv:0902.2796.

[15] C. Michael, Eur. Phys. J. A 31, 793 (2007).

[16] J. Bijnens, P. Gosdzinsky, and P. Talavera, Nucl. Phys. B501, 495 (1997)

[17] L. Del Debbio, L. Giusti, M. Luscher, R. Petronzio, and N. Tantalo, J. High Energy Phys. 02 (2007) 056.

[18] L. Del Debbio, L. Giusti, M. Luscher, R. Petronzio, and N. Tantalo, J. High Energy Phys. 02 (2007) 082.

[19] K. Rummukainen and S. A. Gottlieb, Nucl. Phys. B450, 397 (1995).

[20] C. McNeile and C. Michael (UKQCD), Phys. Lett. B 556, 177 (2003).

[21] S. Aoki et al. (CP-PACS), Phys. Rev. D 76, 094506 (2007).

[22] C. W. Bernard et al., Phys. Rev. D 64, 054506 (2001).

[23] C. Bernard, C.E. Detar, Z. Fu, and S. Prelovsek, Phys. Rev. D 76, 094504 (2007).

[24] C. Aubin et al., Phys. Rev. D 70, 094505 (2004).

[25] E. B. Gregory, A. C. Irving, C. C. McNeile, S. Miller, and Z. Sroczynski, Proc. Sci., LAT2005 (2006) 027 [arXiv: hep-lat/0510066].

[26] M. Luscher, Nucl. Phys. B364, 237 (1991).

[27] C. R. Gattringer and C. B. Lang, Nucl. Phys. B391, 463 (1993).

[28] M. Gockeler, H. A. Kastrup, J. Westphalen, and F. Zimmermann, Nucl. Phys. B425, 413 (1994).

[29] M. Gockeler et al. (QCDSF), arXiv:0810.5337.

[30] S. Durr et al., Science 322, 1224 (2008).

[31] C. Morningstar, arXiv:0810.4448.
[32] B. S. DeWitt, Phys. Rev. 103, 1565 (1956).

[33] R. Frezzotti, P. A. Grassi, S. Sint, and P. Weisz (Alpha), J. High Energy Phys. 08 (2001) 058.

[34] R. Frezzotti and G. C. Rossi, J. High Energy Phys. 08 (2004) 007.

[35] K. Jansen, M. Papinutto, A. Shindler, C. Urbach, and I. Wetzorke (XLF), Phys. Lett. B 619, 184 (2005).

[36] K. Jansen, A. Shindler, C. Urbach, and I. Wetzorke (XLF), Phys. Lett. B 586, 432 (2004).

[37] R. G. Petry, D. Harnett, R. Lewis, and R. M. Woloshyn, Phys. Rev. D 78, 074502 (2008).

[38] A. M. Abdel-Rehim, R. Lewis, and R. M. Woloshyn, Phys. Rev. D 71, 094505 (2005).

[39] A. M. Abdel-Rehim, R. Lewis, R. M. Woloshyn, and J. M. S. Wu, Phys. Rev. D 74, 014507 (2006).

[40] K. Cichy, J. Gonzalez Lopez, K. Jansen, A. Kujawa, and A. Shindler, Nucl. Phys. B800, 94 (2008).

[41] F. Farchioni et al., Eur. Phys. J. C 39, 421 (2005).

[42] F. Farchioni et al., Eur. Phys. J. C 47, 453 (2006).

[43] F. Farchioni et al., Phys. Lett. B 624, 324 (2005).

[44] F. Farchioni et al., Eur. Phys. J. C 42, 73 (2005).

[45] T. Chiarappa et al., Eur. Phys. J. C 50, 373 (2007).

[46] A. Shindler, Phys. Rep. 461, 37 (2008).

[47] P. Boucaud et al. (European Twisted Mass), Phys. Lett. B 650, 304 (2007).

[48] P. Boucaud et al. (European Twisted Mass), Comput. Phys. Commun. 179, 695 (2008).

[49] C. Alexandrou et al. (European Twisted Mass), Phys. Rev. D 78, 014509 (2008).

[50] C. Michael and C. Urbach (European Twisted Mass), Proc. Sci., LAT2007 (2007) 122 [arXiv:0709.4564]

[51] B. Blossier et al. (European Twisted Mass), J. High Energy Phys. 04 (2008) 020.

[52] S. Capitani et al., Phys. Lett. B 639, 520 (2006).

[53] R. Baron et al. (European Twisted Mass), Proc. Sci., LAT2007 (2007) 153 [arXiv:0710.1580].

[54] R. Frezzotti, V. Lubicz, and S. Simula, Phys. Rev. D 79, 074506 (2009).

[55] B. Blossier et al., J. High Energy Phys. 07 (2009) 043.

[56] C. Urbach (European Twisted Mass), Proc. Sci., LAT2007 (2007) 022 [arxiv:0710.1517].

[57] P. Weisz, Nucl. Phys. B212, 1 (1983).

[58] C. Urbach, K. Jansen, A. Shindler, and U. Wenger, Comput. Phys. Commun. 174, 87 (2006).

[59] K. Jansen and C. Urbach, arXiv:0905.3331. 
[60] C. McNeile and C. Michael (UKQCD), Phys. Rev. D 73, 074506 (2006).

[61] R. Frezzotti and G. Rossi, Proc. Sci., LAT2007 (2007) 277 [arXiv:0710.2492].

[62] C. McNeile, C. Michael, and K. J. Sharkey (UKQCD), Phys. Rev. D 65, 014508 (2001).

[63] C. McNeile, C. Michael, and C. Urbach (European Twisted Mass), Phys. Lett. B 674, 286 (2009).

[64] S. Prelovsek, C. Dawson, T. Izubuchi, K. Orginos, and A. Soni, Phys. Rev. D 70, 094503 (2004).

[65] K. Hashimoto and T. Izubuchi, Prog. Theor. Phys. 119, 599 (2008).

[66] C. McNeile and C. Michael (UKQCD), Phys. Rev. D 74, 014508 (2006).

[67] R. Frigori et al., Proc. Sci., LAT2007 (2007) 114 [arXiv:0709.4582].

[68] H.-W. Lin et al. (Hadron Spectrum), Phys. Rev. D 79, 034502 (2009).

[69] K. Jansen, C. Michael, and C. Urbach (European Twisted Mass), Eur. Phys. J. C 58, 261 (2008).

[70] W. M. Yao et al. (Particle Data Group), J. Phys. G 33, 1 (2006).

[71] T. Draper et al., arXiv:0810.5512.

[72] T. A. DeGrand, Phys. Rev. D 43, 2296 (1991).

[73] D. B. Leinweber and T. D. Cohen, Phys. Rev. D 49, 3512 (1994).

[74] D. B. Leinweber, A. W. Thomas, K. Tsushima, and S. V. Wright, Phys. Rev. D 64, 094502 (2001).

[75] C. R. Allton, W. Armour, D. B. Leinweber, A. W. Thomas, and R. D. Young, Phys. Lett. B 628, 125 (2005).

[76] W. Armour, C. R. Allton, D. B. Leinweber, A. W. Thomas, and R. D. Young, J. Phys. G 32, 971 (2006).

[77] C. Allton et al. (RBC and UKQCD), Phys. Rev. D 76, 014504 (2007).

[78] T. DeGrand and C. E. Detar, Lattice Methods for Quantum Chromodynamics (World Scientific, Singapore, 2006), p. 345.

[79] J. Foley et al., Comput. Phys. Commun. 172, 145 (2005).

[80] P. Dimopoulos et al., Proc. Sci., LATTICE2008 (2008) 271 [arXiv:0810.2443].

[81] C. McNeile and C. Michael (UKQCD), Phys. Rev. D 63, 114503 (2001).

[82] E. E. Jenkins, A. V. Manohar, and M. B. Wise, Phys. Rev. Lett. 75, 2272 (1995).

[83] U. G. Meissner, Phys. Rep. 161, 213 (1988).

[84] P. C. Bruns and U.-G. Meissner, Eur. Phys. J. C 40, 97 (2005).

[85] C. Hanhart, J. R. Pelaez, and G. Rios, Phys. Rev. Lett. 100, 152001 (2008).

[86] G. Rios, A. G. Nicola, C. Hanhart, and J.R. Pelaez, arXiv:0905.3489.

[87] S. Aoki et al. (CP-PACS), Phys. Rev. D 60, 114508 (1999).

[88] G. P. Lepage et al., Nucl. Phys. B, Proc. Suppl. 106, 12 (2002).

[89] C. Morningstar, Nucl. Phys. Proc. Suppl. 109A, 185 (2002).

[90] M. R. Schindler and D. R. Phillips, Ann. Phys. (N.Y.) 324, 682 (2009).

[91] Y. Chen et al., arXiv:hep-lat/0405001.

[92] R. Lewis and R. M. Woloshyn, Phys. Rev. D 56, 1571
(1997).

[93] A. Ali Khan et al. (CP-PACS), Phys. Rev. D 65, 054505 (2002).

[94] D. Becirevic, V. Lubicz, F. Mescia, and C. Tarantino, J. High Energy Phys. 05 (2003) 007.

[95] J. Bijnens and P. Gosdzinsky, Phys. Lett. B 388, 203 (1996).

[96] V. Cirigliano and I. Rosell, J. High Energy Phys. 10 (2007) 005.

[97] P. Ball and R. Zwicky, J. High Energy Phys. 04 (2006) 046.

[98] P. Ball, G. W. Jones, and R. Zwicky, Phys. Rev. D 75, 054004 (2007).

[99] V. M. Braun et al., Phys. Rev. D 68, 054501 (2003).

[100] M. Gockeler et al., Proc. Sci., LAT2005 (2006) 063 [arXiv:hep-lat/0509196].

[101] C. Allton et al., Phys. Rev. D 78, 114509 (2008).

[102] P. Dimopoulos et al., Proc. Sci., LAT2007 (2007) 241 [arXiv:0710.0975].

[103] P. Dimopoulos et al. (unpublished).

[104] G. Martinelli, C. Pittori, C. T. Sachrajda, M. Testa, and A. Vladikas, Nucl. Phys. B445, 81 (1995).

[105] J. A. Gracey, Phys. Lett. B 488, 175 (2000).

[106] J. A. Gracey, Nucl. Phys. B662, 247 (2003).

[107] K. G. Chetyrkin, J. H. Kuhn, and M. Steinhauser, Comput. Phys. Commun. 133, 43 (2000).

[108] T. van Ritbergen, J. A. M. Vermaseren, and S. A. Larin, Phys. Lett. B 400, 379 (1997).

[109] M. Czakon, Nucl. Phys. B710, 485 (2005).

[110] M. Gockeler et al., Phys. Rev. D 73, 014513 (2006).

[111] M. Della Morte et al. (ALPHA), Nucl. Phys. B713, 378 (2005).

[112] J. Bijnens, P. Gosdzinsky, and P. Talavera, Phys. Lett. B 429, 111 (1998).

[113] V. Mateu, AIP Conf. Proc. 964, 34 (2007).

[114] O. Cata and V. Mateu, J. High Energy Phys. 09 (2007) 078.

[115] N. Eicker et al. (TXL), Phys. Rev. D 59, 014509 (1998).

[116] P. Ball and V. M. Braun, Phys. Rev. D 58, 094016 (1998).

[117] P. Ball and V. M. Braun, Phys. Rev. D 54, 2182 (1996).

[118] A. P. Bakulev and S. V. Mikhailov, Eur. Phys. J. C 17, 129 (2000).

[119] D. Becirevic and V. Lubicz, Phys. Lett. B 600, 83 (2004).

[120] S. Capitani et al., Nucl. Phys. B, Proc. Suppl. 79, 548 (1999).

[121] O. Cata and V. Mateu, Phys. Rev. D 77, 116009 (2008).

[122] M. V. Chizhov, JETP Lett. 80, 73 (2004).

[123] C. McNeile and C. Michael (UKQCD), Phys. Lett. B 642, 244 (2006).

[124] K. Kawarabayashi and M. Suzuki, Phys. Rev. Lett. 16, 255 (1966).

[125] Riazuddin and Fayyazuddin, Phys. Rev. 147, 1071 (1966).

[126] M. C. Birse, Z. Phys. A 355, 231 (1996).

[127] M. Bando, T. Kugo, and K. Yamawaki, Phys. Rep. 164, 217 (1988).

[128] H. Georgi, Nucl. Phys. B331, 311 (1990).

[129] D. T. Son and M. A. Stephanov, Phys. Rev. D 69, 065020 (2004).

[130] S. Hong, S. Yoon, and M. J. Strassler, J. High Energy Phys. 04 (2006) 003.

[131] V. Bernard, M. Lage, U.-G. Meissner, and A. Rusetsky, J. High Energy Phys. 08 (2008) 024. 